http://revistainvestigacionacademicasinfrontera.com

Recibido el 22 de agosto de 2020. Dictaminado mediante arbitraje favorablemente 18 de noviembre de 2020.

\title{
El impacto del desempeño académico en las carreras de Contador Público, Ingeniería en Administración e Ingeniería en Gestión Empresarial del Instituto Tecnológico de Huatabampo Cohorte generacional 2018.
}

Corral Morales Emma Cecilia: https://orcid.org/0000-0002-0486-117X

Atrip Karam Laila Guadalupe: https://orcid.org/0000-0002-0283-2447

Parra Leyva Martín: https://orcid.org/0000-0001-9907-2140

Gastelum Serrano Jakeline María: https://orcid.org/0000-0002-5173-1167

Tecnológico Nacional de México/ Instituto Tecnológico de Huatabampo.

\section{Resumen}

Esta investigación se realizó con el fin de proponer estrategias para reducir el índice de reprobación escolar, se estudiaron causas de reprobación en cinco fuentes de información; Se analizó el comportamiento de deserción escolar de las carreras de Contador Público, Ingeniería en Administración e Ingeniería en Gestión Empresarial cohorte 2018, se investigó cuáles son los principales factores de deserción en nuestra región y las alternativas para disminuir el índice de reprobación, se detectaron las posibles causas por las cuales los estudiantes reprueban; y se encontró que entre el estudio, familia y trabajo si se siente apoyados por sus padres, el desempeño académico de los estudiantes y docentes es bueno, aun cuando tienen algunas limitantes de servicios.

Palabras Clave: Rendimiento, reprobación, deserción, estrategias

\begin{abstract}
This research was carried out in order to propose strategies to reduce the school failure rate, causes of failure were studied in five sources of information; The behavior of school dropout of the careers of Public Accountant, Engineering in Administration and Engineering in Business Management cohort 2018 was analyzed, the main dropout factors in our region and
\end{abstract}


Año 13.

Núm. 33

Académica sin Frontera

ISSN: 2007-8870

\section{http://revistainvestigacionacademicasinfrontera.com}

Recibido el 22 de agosto de 2020. Dictaminado mediante arbitraje favorablemente 18 de noviembre de 2020.

the alternatives to reduce the failure rate were investigated, Possible causes for which students fail; and it was found that between study, family and work, if they feel supported by their parents, the academic performance of students and teachers is good, even when they have some limitations of services.

Keywords: Performance, disapproval, desertion, strategies.

\section{Introducción}

Se plantea el problema del Alto índice de reprobación de las carreras de ciencias económico administrativas, cohorte generacional 2018. Los antecedentes son en referencia al análisis de la información que arroja el programa de tutorías donde se concentra las calificaciones de los tres primeros semestres, las materias que manejan índice de reprobación y detalla el comportamiento del índice de deserción, entre otros aspectos. El objetivo de los cuestionarios es mejorar el proceso enseñanza - aprendizaje, los factores de estudio son las variables: familiares, académicas, técnicas de estudio, motivación para el estudio, y recurso docente; Están planteados de manera específica para desglosar los pasos que se atendieron para obtener el resultado de los conflictos entre el estudio, familia y trabajo.

La importancia de la investigación sobre rendimiento académico, ayuda al diseño de políticas educativas y estrategias efectivas que guían el desarrollo de la competitividad de los estudiantes para integrarse al mundo productivo. Las investigaciones estudiadas demuestran que las causas de este problema, por lo general no están ligadas solamente a factores relacionados con el plan del sistema escolar ni a las capacidades individuales de las personas, más bien se puede decir que está relacionado a un número importante de factores de origen social, escolar, familiar y cultural; Esto lo encontraron Torres y Rodríguez (2006), en general, señalaron que en su casa respetaban el tiempo en que hacían sus tareas o estudiaban, la mayor 
Año 13.

Núm. 33

Académica sin Frontera

ISSN: 2007-8870

\section{http://revistainvestigacionacademicasinfrontera.com}

Recibido el 22 de agosto de 2020. Dictaminado mediante arbitraje favorablemente 18 de noviembre de 2020.

parte de los encuestados consideraron que los factores más importantes para lograr un mejor aprovechamiento, están relacionados con tener tiempo y un lugar adecuado para estudiar.

La tesis "Factores que influyen en el alto índice de reprobación en las materias de ciencias básicas de la facultad de ingeniería mecánica y eléctrica” escrita por Ing. Juana María Gómez Urrutia, en su análisis concluye que los factores que influyen en al alto índice de reprobación en las materias de Ciencias Básicas según el lugar que ocuparon son:1.- Técnicas de Estudio 2- Motivación para el Estudio 3- Organización para el Estudio 4.- Recursos Académicos Previos 5- Situación Familiar 6.- Recursos Económicos 7- Dinámica Pedagógica 8.Organización de la Clase 9- Horario de Clase y Asesorías 10.- Evaluación del Aprendizaje. La tesis "identificación de los principales factores que influyen en el rendimiento de estudiante de ingeniería industrial y de sistemas en sus primeros semestres" escrita por Roberto Rodolfo Marrufo Pinedo en el año de 2011 en Hermosillo Sonora, concluye que viendo la importancia que tiene el desempeño del estudiante en sus primeros semestres de la carrera, una acción remedial a este problema sería darle un carácter de obligatoriedad a las tutorías, ya que, a la fecha, el alumno si asiste o no, no pasa nada, y creo que, asistiendo, la integración del alumno con sus compañeros y sus maestros será más rápida y su aprendizaje sería más efectivo.

El Tecnológico Nacional de México cuenta con 254 campus distribuidos en todo lo largo y ancho de la República Mexicana; se crea y desincorpora para dar respuesta a las necesidades propias del medio geográfico y social donde se encuentre ubicado, ofertando carreras afines a las necesidades de su región como es el caso del Instituto Tecnológico de Huatabampo que colabora en el crecimiento de una ciudad con alto índice de marginación y pobreza. Se cuenta con un programa de tutorías implementado de manera presencial durante los primeros cuatro semestres. Durante la gestión de las tutorías se obtienen resultados del comportamiento estudiantil en formatos que arrojan información muy enriquecedora para 
Año 13.

Revista de Investigación

Núm. 33

Académica sin Frontera

ISSN: 2007-8870

\section{http://revistainvestigacionacademicasinfrontera.com}

Recibido el 22 de agosto de 2020. Dictaminado mediante arbitraje favorablemente 18 de noviembre de 2020.

lograr un buen análisis del trabajo educativo en cada grupo de alumnos por carrera. Se considera de vital importancia estudiar el índice de reprobación de las carreras ya que en el estudio realizado se detectó que además de contar con un alto índice de deserción por los aspirantes que solo se inscribieron y no asistieron a clase en ningún momento, también se presenta un alto índice de reprobación durante el primer semestre que alcanza el $21 \%$ para Contador Público, 27\% Ingeniería en Administración y 42\% en Ingeniería en Gestión Empresarial. Aunado a eso, el índice de reprobación también ocasiona deserción en un 25\%, $54 \%$ y $33 \%$ respectivamente, porcentaje que se calcula sobre la cantidad de alumnos que reprobaron. Se considera que si se conocen las causas se puede formular un plan de acción para que los alumnos continúen estudiando y mejoren su nivel académico.

Se puede observar en el análisis que el número de estudiantes por grupo en el primer semestre oscila entre los 48 y 36 estudiantes y que después del tercer semestre los grupos quedan integrados por alrededor de 22 alumnos regulares. Que las materias afines y con mayor índice de reprobación durante los primeros semestres son: Contabilidad, costos, algebra, cálculo, estadísticas. También se denota la falta de asesoría por el personal docente ya que las materias que reprueban en el primer semestre esta seriada con una materia afín la cual también se reprueba posteriormente según el comportamiento que arroja la investigación cuantitativa documental.

Se espera que a través de este estudio se logre detectar según el enfoque de los alumnos cuales son los factores que afectan el aprendizaje y con ello poder seleccionar las estrategias idóneas que habrán de atenderse para reducir el índice de reprobación escolar. Porque la responsabilidad de una institución educativa no acaba en la inclusión equitativa sino en la permanencia y cumplimiento de objetivos de que todos cuenten con competencias para ser mejores, específicamente, que los universitarios no sólo se formen como profesionistas, sino que también se eduquen con valores, conocimiento y capacidades 
Año 13.

Núm. 33

Académica sin Frontera

ISSN: 2007-8870

\section{http://revistainvestigacionacademicasinfrontera.com}

Recibido el 22 de agosto de 2020. Dictaminado mediante arbitraje favorablemente 18 de noviembre de 2020. adecuadas para desarrollarse plenamente, con civismo y solidaridad social. Por lo que es preocupante que el Instituto Tecnológico de Huatabampo no nomás no aporte oportunidades de aprendizaje a lo largo de la vida si no que tampoco logre retener al mayor número de aspirantes en sus aulas en una región tan carente de oportunidades, la manifestación del alto índice de reprobación durante los primeros semestres se debe atender en corto plazo.

\section{Material y Método}

El estudio que se describe en cuanto a los antecedentes fue retrospectivo, pues se realizó después de que la cohorte ya se encontraba en 4to semestre, recolectando la evidencia una vez ocurridos los hechos. Se trató de un trabajo con un enfoque cuantitativo de tipo descriptivo y correlacional, como una investigación no experimental, pues se consideraron datos de una muestra que no fue planeada deliberadamente para ello, su enfoque fue longitudinal evolutivo, ya que se analizaron los cambios que se presentaron a través del tiempo. Posteriormente se aplicó el método de investigación mixta para que el problema de la investigación pueda ser aclarado de mejor forma utilizando tanto información cuantitativa como cualitativa. La población que fue estudiada en la cohorte generacional 2018 corresponde a un total de 123 estudiantes tomando una muestra de 45 estudiantes de las tres carreras; Se les envió el link del cuestionario por redes sociales a quienes cuentan con los medios para acceder a un sitio de internet por contingencia sanitaria ya que no se permite salir de casa; cuyas categorías fueron:

La entrevista, desde las encuestas de opinión y las entrevistas abiertas. Un investigador que hace un abordaje desde la perspectiva cualitativa utilizará la entrevista en sus distintas formas de presentarse, pero sus preferencias irán orientadas hacia la entrevista en la que el entrevistado habla de forma abierta. El cuestionario es un conjunto de preguntas diseñadas para generar los datos necesarios para alcanzar los objetivos propuestos del 
Año 13.

Revista de Investigación

Núm. 33

Académica sin Frontera

ISSN: 2007-8870

\section{http://revistainvestigacionacademicasinfrontera.com}

Recibido el 22 de agosto de 2020. Dictaminado mediante arbitraje favorablemente 18 de noviembre de 2020.

proyecto de investigación. Algunos problemas asociados con el envío de los cuestionarios podrían ser: que no fuesen devueltos, los consultados pueden evadir la respuesta y algunas preguntas, o no darles la importancia necesaria a las preguntas proporcionadas. Por ello y otros factores más el instrumento que se use para la recolección de datos debe ser objeto de una cuidadosa elaboración. Las encuestas, la observación, la toma de muestras y las entrevistas, entre otras, permiten realizar la tarea. El trabajo de campo se aplicó las encuestas y cuestionario por Google y solo compartimos el link y esperamos que nos respondieran durante una semana.

\section{Resultados}

\section{Graficas cuestionarios alumnos}

\begin{tabular}{|c|c|c|c|c|}
\hline & SIEMPRE & A VECES & NUNCA & \\
\hline \multirow{2}{*}{$\begin{array}{l}\text { A. AFECTACIONES EN EL ESTUDIO POR } \\
\text { SITUACIONES FAMILIARES }\end{array}$} & $51 \%$ & $38 \%$ & $11 \%$ & \\
\hline & EXCELENTE & BUENO & REGULAR & MALO \\
\hline $\begin{array}{l}\text { B. AFECTACIONES EN EL ESTUDIO POR } \\
\text { SITUACIONES ACADÉMICAS }\end{array}$ & $30 \%$ & $40 \%$ & $26 \%$ & $12 \%$ \\
\hline 1.- Desempeño académico & $18 \%$ & $56 \%$ & $27 \%$ & \\
\hline $\begin{array}{l}\text { 2.- La calidad de los docentes que te impartieron } \\
\text { clases fue: }\end{array}$ & $11 \%$ & $53 \%$ & $36 \%$ & \\
\hline $\begin{array}{l}\text { 3.- La vida académica universitaria (planes, y } \\
\text { programas de estudio) }\end{array}$ & $73 \%$ & $20 \%$ & $7 \%$ & \\
\hline $\begin{array}{l}\text { 4.- La vida académica universitaria (trabajos, } \\
\text { exámenes, etc.) }\end{array}$ & $47 \%$ & $42 \%$ & $11 \%$ & \\
\hline $\begin{array}{l}\text { 5.- Las investigaciones que dejaban realizar } \\
\text { fueron: }\end{array}$ & $31 \%$ & $67 \%$ & $2 \%$ & \\
\hline
\end{tabular}


Año 13.

Revista de Investigación

Académica sin Frontera

Núm. 33

ISSN: 2007-8870

\section{http://revistainvestigacionacademicasinfrontera.com}

Recibido el 22 de agosto de 2020. Dictaminado mediante arbitraje favorablemente 18 de noviembre de 2020.

\begin{tabular}{|c|c|c|c|c|}
\hline $\begin{array}{l}\text { 6.- Tuviste apoyo (orientación, tutoría, o asesorí- } \\
\text { a fuera del salón de clases por parte de tus } \\
\text { profesores }\end{array}$ & $13 \%$ & $4 \%$ & $53 \%$ & $29 \%$ \\
\hline $\begin{array}{l}\text { 7.- El apoyo académico fuera y dentro del salón } \\
\text { de clases por parte de los profesores fue: }\end{array}$ & $16 \%$ & $18 \%$ & $62 \%$ & $4 \%$ \\
\hline 8.- La cantidad de programas en lo semestres: & $7 \%$ & $49 \%$ & $44 \%$ & \\
\hline $\begin{array}{l}\text { 9.- El trabajo dentro y fuera del salón de clases } \\
\text { te resulto: }\end{array}$ & $4 \%$ & $73 \%$ & $22 \%$ & \\
\hline 10.- La carrera que elegiste te resulto: & $44 \%$ & $47 \%$ & $4 \%$ & $4 \%$ \\
\hline $\begin{array}{l}\text { 11.- Los servicios de apoyo académico- } \\
\text { administrativo que el ITHUA proporciona } \\
\text { (biblioteca, librería, servicio médico, orientación } \\
\text { profesional, etc.) te parecieron: }\end{array}$ & $11 \%$ & $53 \%$ & $24 \%$ & $11 \%$ \\
\hline $\begin{array}{l}\text { 12.- El ITHUA en relación a otras instituciones } \\
\text { de Educación Superior es lo que esperabas }\end{array}$ & $82 \%$ & & $18 \%$ & \\
\hline C. TECNICAS DE ESTUDIO & $9 \%$ & $25 \%$ & $56 \%$ & $10 \%$ \\
\hline $\begin{array}{l}\text { 1.- Suele serte difícil seleccionar los puntos más } \\
\text { importantes de los temas de estudia? }\end{array}$ & $2 \%$ & $13 \%$ & $76 \%$ & $11 \%$ \\
\hline $\begin{array}{l}\text { 2.- Sueles tener dificultad en entender tus } \\
\text { apuntes de clase cuando tratas de repasarlos } \\
\text { después de cierto tiempo? }\end{array}$ & $13 \%$ & $20 \%$ & $53 \%$ & $13 \%$ \\
\hline $\begin{array}{l}\text { 3.- ¿Te preparas a veces para un examen } \\
\text { memorizando fórmulas, definiciones o reglas } \\
\text { que no entiendes con claridad? }\end{array}$ & $13 \%$ & $42 \%$ & $38 \%$ & $7 \%$ \\
\hline D. MOTIVACIÓN PARA ESTUDIAR & $46 \%$ & $36 \%$ & $17 \%$ & $10 \%$ \\
\hline $\begin{array}{l}\text { 1.- ¿Crees que, en general basta estudiar lo } \\
\text { necesario para obtener un "aprobado" en las } \\
\text { asignaturas? }\end{array}$ & $20 \%$ & $38 \%$ & $29 \%$ & $11 \%$ \\
\hline
\end{tabular}


Año 13.

Revista de Investigación

Núm. 33

Académica sin Frontera

ISSN: 2007-8870

\section{http://revistainvestigacionacademicasinfrontera.com}

Recibido el 22 de agosto de 2020. Dictaminado mediante arbitraje favorablemente 18 de noviembre de 2020.

\begin{tabular}{|l|l|l|l|l|}
\hline $\begin{array}{l}\text { 2.- ¿Sueles pensar que no vale la pena el tiempo } \\
\text { y el esfuerzo que son necesarios para lograr una } \\
\text { educación universitaria? }\end{array}$ & $84 \%$ & $16 \%$ & & \\
\hline $\begin{array}{l}\text { 3- ¿Crees que es más importante divertirte y } \\
\text { disfrutar de la vida que estudiar? }\end{array}$ & $62 \%$ & $36 \%$ & $2 \%$ & \\
\hline $\begin{array}{l}\text { 4.- ¿Sientes frecuentes deseos de abandonar la } \\
\text { universidad y conseguir un trabajo? }\end{array}$ & $56 \%$ & $40 \%$ & $4 \%$ & $9 \%$ \\
\hline $\begin{array}{l}\text { 5.- ¿Esperas normalmente a que se te fije la fecha } \\
\text { de un examen para comenzar a estudiar los libros } \\
\text { de texto o de repasar tus apuntes de clase? }\end{array}$ & $9 \%$ & $51 \%$ & $31 \%$ & $\mathbf{3 \%}$ \\
\hline E. RECURSO DOCENTE & $\mathbf{3 4 \%}$ & $\mathbf{4 7 \%}$ & $\mathbf{2 3 \%}$ & \\
\hline $\begin{array}{l}\text { 1.- ¿Los profesores faltan raras veces y cuando } \\
\text { lo hacen avisan con anterioridad? }\end{array}$ & $38 \%$ & $62 \%$ & & \\
\hline $\begin{array}{l}\text { 2.- ¿Los profesores establecen horario de } \\
\text { consulta y cumplen con dicho horario? }\end{array}$ & $11 \%$ & $53 \%$ & $31 \%$ & \\
\hline $\begin{array}{l}\text { 3.- ¿Los profesores presentan desde el principio } \\
\text { un programa completo de la materia? }\end{array}$ & $49 \%$ & $40 \%$ & $11 \%$ & \\
\hline $\begin{array}{l}\text { 4.- ¿Provee suficiente bibliografía y elementos } \\
\text { de consulta? }\end{array}$ & $27 \%$ & $29 \%$ & $44 \%$ & \\
\hline $\begin{array}{l}\text { 5.- ¿Comienza las clases demostrando la } \\
\text { importancia e interés del tema? }\end{array}$ & $44 \%$ & $49 \%$ & \\
\hline
\end{tabular}

Los hallazgos en la encuesta del estudiante que arrojaron las variables entre el estudio, familia y trabajo, demuestra que la mayoría de los padres no son profesionistas y si apoyan los estudios de sus hijos porque se considera que los problemas familiares si tiene reflejo en las actividades escolares; El desempeño académico de los estudiantes y docentes es bueno, también están satisfechos con la carrera elegida y el nivel académico del ITHua, aun cuando no están satisfechos con el ambiente estudiantil, falta servicio médico, orientación 
Año 13.

Revista de Investigación

Núm. 33

Académica sin Frontera

ISSN: 2007-8870

\section{http://revistainvestigacionacademicasinfrontera.com}

Recibido el 22 de agosto de 2020. Dictaminado mediante arbitraje favorablemente 18 de noviembre de 2020.

profesional y asesoría docente; En las técnicas de estudio les resulta difícil estudiar de sus apuntes y seleccionar los puntos más importantes para estudiar para un examen; en cuanto a la motivación para estudiar es solo cuando se presenta el periodo de examen consideran de mucha importancia terminar su carrera, pero si han pensado abandonar sus estudios y buscar un empleo.

\section{Graficas encuestas maestros}

\begin{tabular}{|c|c|c|c|}
\hline & $\begin{array}{l}\text { TOTALMENTE } \\
\text { DE ACUERDO }\end{array}$ & $\begin{array}{l}\text { DE } \\
\text { ACUERDO }\end{array}$ & $\begin{array}{l}\text { EN } \\
\text { DESACUERD } \\
0\end{array}$ \\
\hline SITUACIÓN FAMILIAR & $50 \%$ & $50 \%$ & 0 \\
\hline $\begin{array}{l}\text { 1.- Cree usted que los padres están al pendiente de } \\
\text { sus hijos }\end{array}$ & $20 \%$ & $80 \%$ & $0 \%$ \\
\hline $\begin{array}{l}\text { 2.- cree que los problemas familiares perjudiquen } \\
\text { al rendimiento académico de los alumnos }\end{array}$ & $80 \%$ & $20 \%$ & \\
\hline FACTORES ATRIBUIDOS AL ALUMNO & $30 \%$ & $56 \%$ & $14 \%$ \\
\hline 1.- Falta de interés & $60 \%$ & $40 \%$ & \\
\hline 2.- Falta de conocimientos previos a la materia & $40 \%$ & $60 \%$ & \\
\hline 3.- Falta de disciplina para el estudio & $60 \%$ & $40 \%$ & \\
\hline 4.- Deficiencia en métodos de estudio & & $60 \%$ & $40 \%$ \\
\hline 5.- Falta de motivación & & $60 \%$ & $40 \%$ \\
\hline 6.- Falta de apoyo moral familiar & & $80 \%$ & $20 \%$ \\
\hline 7.- Falta de apoyo económico & $20 \%$ & $40 \%$ & $40 \%$ \\
\hline 8.- Falta de metas claras y definidas & $20 \%$ & $80 \%$ & \\
\hline 9.- Falta de responsabilidad & $60 \%$ & $40 \%$ & \\
\hline 10.- Actitudes negativas & $40 \%$ & $60 \%$ & \\
\hline $\begin{array}{l}\text { FACTORES ATRIBUIDOS A LOS } \\
\text { PROFESORES }\end{array}$ & $11 \%$ & $40 \%$ & $49 \%$ \\
\hline 1.- Falta de responsabilidad en horario de clase & $20 \%$ & $40 \%$ & $40 \%$ \\
\hline $\begin{array}{l}\text { 2.- Falta de organización en la preparación de la } \\
\text { clase }\end{array}$ & $20 \%$ & $40 \%$ & $40 \%$ \\
\hline 3.- Falta de motivación en la enseñanza & & $60 \%$ & $40 \%$ \\
\hline $\begin{array}{l}\text { 4.- Falta de conocimientos en la materia que } \\
\text { imparte }\end{array}$ & $20 \%$ & $40 \%$ & $40 \%$ \\
\hline
\end{tabular}


Año 13.

Revista de Investigación

Núm. 33

Académica sin Frontera

ISSN: 2007-8870

\section{http://revistainvestigacionacademicasinfrontera.com}

Recibido el 22 de agosto de 2020. Dictaminado mediante arbitraje favorablemente 18 de noviembre de 2020.

\begin{tabular}{|l|l|l|l|}
\hline $\begin{array}{l}\text { 5.- Falta de capacidad para transmitir } \\
\text { conocimientos }\end{array}$ & $20 \%$ & $40 \%$ & $40 \%$ \\
\hline 6.- Forma de evaluar el aprendizaje & & $40 \%$ & $60 \%$ \\
\hline 7.- Relación con el estudiante & & $20 \%$ & $80 \%$ \\
\hline $\begin{array}{l}\text { FACTORES ATRIBUIDOS A LA } \\
\text { INSTITUCIÓN }\end{array}$ & $\mathbf{5 0 \%}$ & $\mathbf{5 0 \%}$ \\
\hline \begin{tabular}{l} 
1.- Planes de estudio inalcanzables \\
\hline $\begin{array}{l}\text { 2.- Infraestructura inadecuada (salón, biblioteca, } \\
\text { laboratorios etc.) }\end{array}$
\end{tabular} & $60 \%$ & $100 \%$ \\
\hline $\begin{array}{l}\text { 3.- Perfil del alumno de nuevo ingreso } \\
\text { inadecuado }\end{array}$ & $100 \%$ & $40 \%$ \\
\hline $\begin{array}{l}\text { 4.- Hace falta más comunicación entre } \\
\text { MAESTRO-ALUMNO }\end{array}$ & $40 \%$ & $60 \%$ \\
\hline
\end{tabular}

En la encuesta de opinión con los coordinadores de carrera, una variable fue el comportamiento del estudiante y se detectó: la falta de interés, bajo nivel de conocimiento, falta de disciplina, necesidad de método de estudio, no tienen metas claras, poca responsabilidad y una actitud negativa. Otra variable fue el comportamiento docente donde la opinión fue: la falta de capacidad para trasmitir conocimientos y fortalecer una buena relación con los estudiantes.

Se puede observar la carencia de la aplicación pedagógica, falta seguimiento al estudiante a través de asesorías, implementar correctamente el programa de tutorías y crear un ambiente estudiantil que aumente la motivación con la participación docente.

\section{Discusión}

La definición del rendimiento académico enmarca las limitaciones que intervienen en la interiorización de conocimientos de acuerdo a un perfil establecido, la reprobación, es un término que se utiliza para etiquetar a quienes no lograron obtener el puntaje mínimo que les acredita el aprendizaje de los conocimientos esperados planteados por el plan de estudios (Gutiérrez y Montañez, 2012). El nivel de logro, en relación a lo que contempla el currículo 
Año 13.

Núm. 33

Académica sin Frontera

ISSN: 2007-8870

\section{http://revistainvestigacionacademicasinfrontera.com}

Recibido el 22 de agosto de 2020. Dictaminado mediante arbitraje favorablemente 18 de noviembre de 2020.

nacional, debe precisar los niveles de desempeño que todos los alumnos deben adquirir y las pruebas son los instrumentos que cumplen con la función de determinar si los alumnos cuentan o no con el dominio del conocimiento brindado por la escuela y establecidos en los programas vigentes.

De una manera más completa el diccionario de las ciencias de educación (1995), define al rendimiento escolar como el nivel de conocimiento de un alumno medido con una evaluación y que además éste modulado por diversos factores psicológicos, sociológicos, pedagógicos, entre otros más. Una de sus características principales es que se lleva a través de un proceso, que contempla tres etapas: la evaluación de inicio o diagnóstica, ésta ayuda a conocer los saberes previos de los estudiantes, la evaluación formativa que se produce durante el proceso de enseñanza y la evaluación sumativa cuyo fin es tomar decisiones para la acreditación. La evaluación es parte fundamental del proceso de enseñanza-aprendizaje, por tal motivo, no se puede considerar como un elemento aislado, ya que está en conexión con el resto de las partes que integran dicho proceso. La evaluación relacionada al campo de la educación, puede depender de diferentes aspectos, entre los cuales podemos nombrar: el sistema educativo, la administración escolar, el personal docente, las instalaciones y otros más; en base a estos aspectos, se puede estimar utilizando diversos criterios como la utilidad, rendimiento, eficiencia, etc. (Carreño, citado por Zuñiga, 1989). Aunque los exámenes no son los únicos instrumentos de evaluación, son los utilizados por las autoridades educativas nacionales e internacionales para determinar los niveles de logro de cada niño, éste tipo de evaluación (cuantitativas), contradice lo establecido por los planes y programas actuales basados en competencias, los cuales establecen que la evaluación debe estar conformada con las calificaciones de diferentes saberes, tales como: aprender a hacer, aprender ser, aprender a conocer y aprender a convivir, esto significa que la evaluación no debe ser únicamente 
Año 13.

Núm. 33

Académica sin Frontera

ISSN: 2007-8870

\section{http://revistainvestigacionacademicasinfrontera.com}

Recibido el 22 de agosto de 2020. Dictaminado mediante arbitraje favorablemente 18 de noviembre de 2020.

cuantitativa sino que además debe ser cualitativa. Todo lo que el resultado se compare con otras investigaciones.

Por otra parte, está la perspectiva del estudiante: En el contexto escolar los profesores valoran más el esfuerzo que la habilidad. En otras palabras, mientras un estudiante espera ser reconocido por su capacidad (lo cual resulta importante para su estima), en el salón de clases se reconoce su esfuerzo. De acuerdo con lo anterior se derivan tres tipos de estudiantes según Covington (1984):

a. "Los orientados al dominio. Sujetos que tienen éxito escolar, se consideran capaces, presentan alta motivación de logro y muestran confianza en sí mismos.

b. Los que aceptan el fracaso. Sujetos derrotistas que presentan una imagen propia deteriorada y manifiestan un sentimiento de desesperanza aprendido, es decir que han aprendido que el control sobre el ambiente es sumamente difícil o imposible, y por lo tanto renuncian al esfuerzo.

c. Los que evitan el fracaso. Aquellos estudiantes que carecen de un firme sentido de aptitud y autoestima y ponen poco esfuerzo en su desempeño; para "proteger" su imagen ante un posible fracaso, recurren a estrategias como la participación mínima en el salón de clases, retraso en la realización de una tarea, trampas en los exámenes, etc. "

En éste orden de ideas, el juego de valores habilidad-esfuerzo se torna riesgoso para los alumnos, ya que, si tienen éxito, decir que se invirtió poco o nada de esfuerzo implica brillantez, esto es, se es muy hábil. Cuando se invierte mucho esfuerzo no se ve el verdadero nivel de habilidad, de tal forma que esto no amenaza la estima o valor como estudiante, y en tal caso, el sentimiento de orgullo y la satisfacción son grandes.

Lo anterior significa que, en una situación de éxito, las autopercepciones de habilidad y esfuerzo no perjudican ni dañan la estima ni el valor que el profesor otorga. Sin embargo, 
Año 13.

Revista de Investigación

Núm. 33

Académica sin Frontera

ISSN: 2007-8870

\section{http://revistainvestigacionacademicasinfrontera.com}

Recibido el 22 de agosto de 2020. Dictaminado mediante arbitraje favorablemente 18 de noviembre de 2020.

cuando la situación es de fracaso, las cosas cambian. Decir que se invirtió gran esfuerzo implica poseer poca habilidad, lo que genera un sentimiento de humillación. Así el esfuerzo empieza a convertirse en un arma de doble filo y en una amenaza para los estudiantes, ya que éstos deben esforzarse para evitar la desaprobación del profesor, pero no demasiado, porque en caso de fracaso, sufren un sentimiento de humillación e inhabilidad. Dado que una situación de fracaso pone en duda su capacidad, es decir, su autovaloración, algunos estudiantes evitan este riesgo, y para ello emplean ciertas estrategias como la excusa y manipulación del esfuerzo, con el propósito de desviar la implicación de inhabilidad (Covington y Omelich, 1979).

Como se menciona, algunas de las estrategias pueden ser: tener una participación mínima en el salón de clases (no se fracasa pero tampoco se sobresale), demorar la realización de una tarea (el sujeto que estudia una noche antes del examen: en caso de fracaso, este se atribuye a la falta de tiempo y no de capacidad), no hacer ni el intento de realizar la tarea (el fracaso produce menos pena porque esto no es sinónimo de incapacidad), el sobreesfuerzo, el copiar en los exámenes y la preferencia de tareas muy difíciles (si se fracasa, no estuvo bajo el control del sujeto), o muy fáciles (de tal manera que aseguren el éxito). En otras palabras, se fracasa con "honor" por la ley del mínimo esfuerzo.

El empleo desmedido de estas estrategias trae como consecuencia un deterioro en el aprendizaje, se está propenso a fracasar y se terminará haciéndolo tarde o temprano, lo que en forma análoga nos recuerda el "efecto Pigmalión" en el proceso educativo, es decir, una profecía de fracaso escolar que es autocumplida. Resulta evidente, que el abordaje del rendimiento académico no podría agotarse a través del estudio de las percepciones de los alumnos sobre las variables habilidad y esfuerzo, así como tampoco podría ser reducida a la simple comprensión entre actitud y aptitud del estudiante. 


\section{http://revistainvestigacionacademicasinfrontera.com}

Recibido el 22 de agosto de 2020. Dictaminado mediante arbitraje favorablemente 18 de noviembre de 2020.

\section{Conclusiones}

Se analizó el comportamiento de reprobación escolar de las carreras de Contador Público, Ingeniería en Administración e Ingeniería en Gestión Empresarial cohorte 2018, se investigaron cuáles son los principales factores de deserción en nuestra región y se detectó que las causas por las cuales los estudiantes del área de ciencias económico Administrativas reprueban por conflictos entre el estudio, familia y trabajo se debe a la falta de seguimiento y detección oportuna de estudiantes con necesidades de asesoría docente tanto en conocimiento como en las técnicas de estudio. Que esto impacta en la motivación ya que el estudiante no recibe atención oportuna y cada vez es mayor la cantidad de materias que reprueba sin encontrar respuesta a su problema. Las alternativas para disminuir el índice de reprobación que arroja la investigación documental que mayor impacto tendría en las carreras de ciencias económico administrativas son: Dar a conocer y realizar prácticas con los estudiantes de las técnicas de estudio y fortalecer en el docente las técnicas pedagógicas para lograr mejores resultados en el aula y promover eventos de integración estudiante maestro. La mejor alternativa educativa para aumentar la preparación escolar de los conocimientos adquiridos en escuelas de educación media superior es implementar el curso de nivelación o propedéutico. Proponer alternativas de cambio de distribución de la retícula por las academias correspondientes que hagan más amigables las cargas académicas durante los primeros semestres. Es claro que si los estudiantes reprueban es porque hay falta de interés y compromiso hacen las cosas nada más por hacerlas y no tienen metas claras a futuro. También en ocasiones el profesor no es apto para la materia que está impartiendo y esto hace que los estudiantes se distraigan y deserten. Definitivamente el alumno se prepara para un examen hasta el último momento, deja para el último momento la preparación de sus trabajos, no entiende sus apuntes cuando trata de estudiarlos debido al tiempo que dejo pasar, estudia memorizando únicamente, sin entender ni mucho menos comprender. 


\section{Año 13.}

Revista de Investigación

Núm. 33

\section{Académica sin Frontera}

ISSN: 2007-8870

\section{http://revistainvestigacionacademicasinfrontera.com}

Recibido el 22 de agosto de 2020. Dictaminado mediante arbitraje favorablemente 18 de noviembre de 2020.

Ante todo, lo anterior se pueden atender dos cosas primero mejorar la manera de evaluarlos que pudiera ser más seguida, por temas o por unidades de tal manera que se tengan alumno estudiando continuamente. Y segundo que se tenga un departamento especializado en asesoría de técnicas de estudio y conocimiento, que realmente se cumpla con el horario por parte tanto de maestros como de alumnos, que el maestro tutor cuando detecte estudiantes con necesidad de asesoría lo canalice y le dé seguimiento para nivelar el grupo. Se debe programar y vigilar la optimización de las horas de asesorías seleccionando los maestros asesores con perfil pedagógico.

\section{Agradecimientos}

A la Ing. Guadalupe Barreras Álvarez, Jefa del Departamento de Servicios Escolares del ITHua por su invaluable disposición al proporcionar la información de manera precisa y oportuna.

A los estudiantes que dieron respuesta a las encuestas con veracidad y amplio criterio.

\section{Referencias}

1. ANUIES - SEP, Comités Interinstitucionales para la Evaluación de la Educación Superior, Marco de referencia para la evaluación, Comité de Ingeniería y Tecnología, Octubre de 1994, México.

2. Camarena C. Rosa María, Chávez G. Ana María y Gómez V. José, Reflexiones en torno al rendimiento escolar, Editorias Hugar, Septiembre 1985, México, Brown, W. F. \& TRILLAS, EDITORIAL. (2010). Guia Para La Supervivencia Del Estudiante (3.a ed.). Trillas.

3. Escudero Yerena, María Teresa, E. Y. M. T. (1997). La comunicación en la enseñanza. Editorial Trillas. 
Año 13.

Revista de Investigación

Núm. 33

Académica sin Frontera

ISSN: 2007-8870

http://revistainvestigacionacademicasinfrontera.com

Recibido el 22 de agosto de 2020. Dictaminado mediante arbitraje favorablemente 18 de noviembre de 2020.

4. Edel Navarro, Rubén (2003). "El rendimiento académico: concepto, investigación y desarrollo", Revista Electrónica Iberoamericana sobre Calidad, Eficacia y Cambio en

Educación, $\quad$ vol. $1, \quad$ núm. $\quad 2 . \quad$ Disponible http://www.ice.deusto.es/rinace/reice/vol1no2/Edel.pdf.

5. González Lomelí, Daniel (2002). El desempeño académico universitario: variables psicológicas asociadas. Hermosillo, Sonora: PROMEP-UniSon

6. Ibabe Erostarbe, Izaskun y Jaureguizar Albonigamayor, Joana. (2007). "Autoevaluación a través de Internet: variables metacognitivas y rendimiento académico", Revista Latinoamericana de Tecnología Educativa, 6 (2). Disponible en http://campusvitual.unex.es/cala/editio.

\section{Apéndice}

ENCUESTA DIRIGIDO A LOS JEFES ACADEMICOS Y DOCENTES DEL AREA ECONOMICO ADMINISTRATIVO PARA DETECTAR POSIBLES FACTORES QUE INFLUYEN EN LOS NIVELES DE REPROBACION DEL AREA ECONOMICO ADMINISTRATIVO DEL INSTITUTO TECNOLOGICO DE HUATABAMPO. OBJETIVO DEL CUESTIONARIO:

Su información es valiosa ya que ayudara a mejorar el proceso enseñanza - aprendizaje o cualquier factor que influya en tan grave problema " la reprobación".

Instrucciones:

Marque la respuesta que contesta más acertadamente a la pregunta. Solo una respuesta por pregunta.

A) ( ) JEFE ACADEMICO B) ( ) PROFESOR

CARRERA: A) C.P B) IGE C) I.A

I.- SITUACIONES FAMILIARES 


\section{http://revistainvestigacionacademicasinfrontera.com}

Recibido el 22 de agosto de 2020. Dictaminado mediante arbitraje favorablemente 18 de noviembre de 2020.

1.- Cree usted que los padres estén al pendiente de sus hijos

A) ( ) Totalmente de acuerdo B) ( ) de acuerdo C) ( ) en desacuerdo D) ( ) indiferente

2.- cree que los problemas familiares perjudiquen al rendimiento académico de los alumnos

A) ( ) Totalmente de acuerdo B) ( ) de acuerdo C) ( ) en desacuerdo D) ( ) indiferente

II.- FACTORES ATRIBUIDOS AL ALUMNO

1.- Falta de interés

A) ( ) Totalmente de acuerdo B) ( ) de acuerdo C) ( ) en desacuerdo D) ( ) indiferente

2.- Falta de conocimientos previos a la materia

A) ( ) Totalmente de acuerdo B) ( ) de acuerdo C) ( ) en desacuerdo D) ( ) indiferente 3.- Falta de disciplina para el estudio

A) ( ) Totalmente de acuerdo B) ( ) de acuerdo C) ( ) en desacuerdo D) ( ) indiferente 4.- Deficiencia en métodos de estudio

A) ( ) Totalmente de acuerdo B) ( ) de acuerdo C) ( ) en desacuerdo D) ( ) indiferente 5.- Falta de motivación

A) ( ) Totalmente de acuerdo B) ( ) de acuerdo C) ( ) en desacuerdo D) ( ) indiferente 6.- Falta de apoyo moral familiar

A) ( ) Totalmente de acuerdo B) ( ) de acuerdo C) ( ) en desacuerdo D) ( ) indiferente 7.- Falta de apoyo económico

A) ( ) Totalmente de acuerdo B) ( ) de acuerdo C) ( ) en desacuerdo D) ( ) indiferente 8.- Falta de metas claras y definidas

A) ( ) Totalmente de acuerdo B) ( ) de acuerdo C) ( ) en desacuerdo D) ( ) indiferente 9.- Falta de responsabilidad

A) ( ) Totalmente de acuerdo B) ( ) de acuerdo C) ( ) en desacuerdo D) ( ) indiferente 10.- Actitudes negativas

A) ( ) Totalmente de acuerdo B) ( ) de acuerdo C) ( ) en desacuerdo D) ( ) indiferente 


\section{http://revistainvestigacionacademicasinfrontera.com}

Recibido el 22 de agosto de 2020. Dictaminado mediante arbitraje favorablemente 18 de noviembre de 2020 .

\section{III.- FACTORES ATRIBUIDOS A LOS PROFESORES}

1.- Falta de responsabilidad en horario de clase

A) ( ) Totalmente de acuerdo B) ( ) de acuerdo C) ( ) en desacuerdo D) ( ) indiferente 2.- Falta de organización en la preparación de la clase

A) ( ) Totalmente de acuerdo B) ( ) de acuerdo C) ( ) en desacuerdo D) ( ) indiferente 3.- Falta de motivación en la enseñanza

A) ( ) Totalmente de acuerdo B) ( ) de acuerdo C) ( ) en desacuerdo D) ( ) indiferente 4.- Falta de conocimientos en la materia que imparte

A) ( ) Totalmente de acuerdo B) ( ) de acuerdo C) ( ) en desacuerdo D) ( ) indiferente 5.- Falta de capacidad para transmitir conocimientos

A) ( ) Totalmente de acuerdo B) ( ) de acuerdo C) ( ) en desacuerdo D) ( ) indiferente 6.- Forma de evaluar el aprendizaje

A) ( ) Totalmente de acuerdo B) ( ) de acuerdo C) ( ) en desacuerdo D) ( ) indiferente 7.- Relación con el estudiante

A) ( ) Totalmente de acuerdo B) ( ) de acuerdo C) ( ) en desacuerdo D) ( ) indiferente IV.- FACTORES ATRIBUIDOS A LA INSTITUCION

1.- Planes de estudio inalcanzables

A) ( ) Totalmente de acuerdo B) ( ) de acuerdo C) ( ) en desacuerdo D) ( ) indiferente 2.- Infraestructura inadecuada (salón, biblioteca, laboratorios etc.)

A) ( ) Totalmente de acuerdo B) ( ) de acuerdo C) ( ) en desacuerdo D) ( ) indiferente 3.- Perfil del alumno de nuevo ingreso inadecuado

A) ( ) Totalmente de acuerdo B) ( ) de acuerdo C) ( ) en desacuerdo D) ( ) indiferente 4.- Hace falta más comunicación entre MAESTRO-ALUMNO

A) ( ) Totalmente de acuerdo B) ( ) de acuerdo C) ( ) en desacuerdo D) ( ) indiferente 
Año 13.

Revista de Investigación

Núm. 33

Académica sin Frontera

ISSN: 2007-8870

\section{http://revistainvestigacionacademicasinfrontera.com}

Recibido el 22 de agosto de 2020. Dictaminado mediante arbitraje favorablemente 18 de noviembre de 2020.

Mencione otras causas por las que usted crea que exista un alto Índice de reprobación en el ITHUA.

CUESTIONARIO PARA EL ANALISIS DE CAUSAS DE REPROBACIÓN DE LOS

ESTUDIANTES DEL INSTITUTO TECNOLOGICO DE HUATABAMPO DE LAS CARRERAS ECONOMICO ADMINISTRATIVO CONTADOR PUBLICO, INGENIERA EN ADMINISTRACION E INGENIERA EN GESTION EMPRESARIAL COHORTE 2018 .

NOMBRE:

SEMESTRE: A) 2do $\quad$ B) 4to $\quad$ C) Otro

$\begin{array}{lll}\text { CARRERA: A) C.P } & \text { B) IGE } & \text { C) I.A }\end{array}$

NUMERO DE CONTROL:

OBJETIVO DEL CUESTIONARIO:

Tu información es valiosa ya que ayudara a mejorar el proceso enseñanza - aprendizaje o cualquier factor que influya en tan grave problema " la reprobación".

INSTRUCCIONES:

En el espacio de la izquierda anota una $X$ en la opción que consideres más adecuada. Todas las preguntas son en referencia a los semestres anteriores.

A) SITUACIONES FAMILIARES

1.-Tus padres trabajan desarrollando alguna profesión
A) ( ) SI
B) ( ) $\mathrm{NO}$

2.-Están al pendiente de tus estudios al menos uno de tus padres
A) ( ) Siempre B) ( ) casi siempre
C) ( ) a veces
D) ( ) nunca

3.-Los problemas familiares te perjudican en el estudio
A) ( ) Siempre
B) ( ) casi siempre
C) ( ) a veces
D) ( ) nunca
B) VARIABLES ACADEMICAS 
Año 13.

Revista de Investigación

Núm. 33

Académica sin Frontera

ISSN: 2007-8870

\section{http://revistainvestigacionacademicasinfrontera.com}

Recibido el 22 de agosto de 2020. Dictaminado mediante arbitraje favorablemente 18 de noviembre de 2020.

1.- Tu desempeño académico fue:

A) ( ) Excelente B) ( ) bueno C) ( ) regular D) ( ) deficiente E) ( ) muy deficiente

2.- La calidad de los docentes que te impartieron clases fue:

A) ( ) Excelente B) ( ) bueno C) ( ) regular D) ( ) deficiente E) ( ) muy deficiente

3.- La vida académica universitaria (planes, y programas de estudio)

A) ( ) Te resulto muy pesada B) ( ) te resulto pesada C) ( ) te resulto adecuada D) ( ) te resulto ligera

4.-La vida académica universitaria (trabajos, exámenes, etc.)

A) ( ) Te resulto muy pesada B) ( ) te resulto pesada C) ( ) te resulto adecuada D) ( ) te resulto ligera

5.- Las investigaciones que dejaban realizar fueron:

A) ( ) De muy alto nivel B) ( ) de alto nivel C) ( ) de regular nivel D) ( ) bajo nivel ( ) de muy bajo nivel

6.- Tuviste apoyo (orientación, tutoría, o asesoría) fuera del salón de clases por parte de tus profesores

A) ( ) Siempre B) ( ) casi siempre C) ( ) a veces D) ( ) nunca

7.-El apoyo académico fuera y dentro del salón de clases por parte de los profesores fue:

A) ( ) De excelente calidad B) ( ) de mucha calidad C) ( ) de calidad D) ( ) De poca calidad 8.- La cantidad de programas en lo semestres:

A) ( ) Nunca daba tiempo de cubrirlos B) ( ) se cubrían apretadamente C) ( ) se cubrían en el tiempo establecido D) ( ) se cubrían ampliamente E) ( ) se cubrían y sobraba tiempo 9.- El trabajo dentro y fuera del salón de clases te resulto:

A) ( ) Muy pesado B) ( ) pesado C) ( ) razonable D) ( ) ligero E) ( ) muy ligero

10.- La carrera que elegiste te resulto: 
Año 13.

Revista de Investigación

Núm. 33

Académica sin Frontera

ISSN: 2007-8870

\section{http://revistainvestigacionacademicasinfrontera.com}

Recibido el 22 de agosto de 2020. Dictaminado mediante arbitraje favorablemente 18 de noviembre de 2020.

A) ( ) Muy interesante B) ( ) interesante C) ( ) medianamente interesante D) ( ) poco interesante E) ( ) no era lo que esperabas

11.- los servicios de apoyo académico-administrativo que el ITHUA proporciona (biblioteca, librería, servicio médico, orientación profesional, etc.) te parecieron:

A) ( ) Muy buenos B) ( ) Buenos C) ( ) Satisfactorias D) ( ) malos E) ( ) muy malos

12.- el ITHUA en relación a otras instituciones de Educación Superior es lo que esperabas

A) ( ) SI B) ( ) NO

C) EN CUANTO A TECNICAS DE ESTUDIO

1.- ¿Suele serte difícil seleccionar los puntos más importantes de los temas de estudia?

A ( ) Muy pesado B) ( ) pesado C) ( ) razonable D) ( ) ligero E) ( ) muy ligero

2.- ¿Sueles tener dificultad en entender tus apuntes de clase cuando tratas de repasarlos después de cierto tiempo?

A ( ) Muy pesado B) ( ) pesado C) ( ) razonable D) ( ) ligero E) ( ) muy ligero

3.- ¿Te preparas a veces para un examen memorizando fórmulas, definiciones o reglas que no entiendes con claridad?

A) ( ) Siempre B) ( ) casi siempre C) ( ) a veces D) ( ) nunca

D) EN CUANTO A MOTIVACION PARA EL ESTUDIO

1.- ¿Crees que, en general basta estudiar lo necesario para obtener un "aprobado" en las asignaturas?

A) ( ) Siempre B) ( ) casi siempre C) ( ) a veces D) ( ) nunca

2.- ¿Sueles pensar que no vale la pena el tiempo y el esfuerzo que son necesarios para lograr una educación universitaria?

A) ( ) Siempre B) ( ) casi siempre C) ( ) a veces D) ( ) nunca

3- ¿Crees que es más importante divertirte y disfrutar de la vida que estudiar?

A) ( ) Totalmente de acuerdo B) ( ) de acuerdo C) ( ) en desacuerdo D) ( ) indiferente 
Año 13.

Revista de Investigación

Núm. 33

\section{http://revistainvestigacionacademicasinfrontera.com}

Recibido el 22 de agosto de 2020. Dictaminado mediante arbitraje favorablemente 18 de noviembre de 2020.

4.- ¿Sientes frecuentes deseos de abandonar la universidad y conseguir un trabajo?

A) ( ) Siempre B) ( ) casi siempre C) ( ) a veces D) ( ) nunca

5.- ¿Esperas normalmente a que se te fije la fecha de un examen para comenzar a estudiar los libros de texto o de repasar tus apuntes de clase?

A) ( ) Siempre B) ( ) casi siempre C) ( ) a veces D) ( ) nunca

E) RECURSOS DOCENTES

1.- ¿Los profesores faltan raras veces y cuando lo hacen avisan con anterioridad?

A) La mayoría B) pocos C) ninguno D) otro

2.- ¿Los profesores establecen horario de consulta y cumplen con dicho horario?

A) ( ) Siempre B) ( ) casi siempre C) ( ) a veces D) ( ) nunca

3.- ¿Los profesores presentan desde el principio un programa completo de la materia?

A) ( ) Siempre B) ( ) casi siempre C) ( ) a veces D) ( ) nunca

4.- ¿Provee suficiente bibliografía y elementos de consulta?

A) ( ) Siempre B) ( ) casi siempre C) ( ) a veces D) ( ) nunca

5.- ¿Comienza las clases demostrando la importancia e interés del tema?

( ) Siempre ( ) casi siempre ( ) a veces ( ) nunca 
"Fl whiser ite mis hijos

Año 13.
Revista de Investigación

Académica sin Frontera

ISSN: 2007-8870

http://revistainvestigacionacademicasinfrontera.com

Recibido el 22 de agosto de 2020. Dictaminado mediante arbitraje favorablemente 18 de noviembre de 2020.

\section{Directorio Institucional}

Dr. Enrique Fernando Velázquez Contreras

Rector

Dr. Ramón Enrique Robles Zepeda

Secretario General Académico

Dra. Rosa María Montesinos Cisneros

Secretaria General Administrativa

Dr. Rodolfo Basurto Álvarez

Director de Vinculación y Difusión

Dra. Adriana Leticia Navarro Verdugo

Vicerrectora de la Unidad Regional Sur

Dr. Ernesto Clark Valenzuela

Director de la División de Ciencias Económicas y Sociales

Dr. Francisco Espinoza Morales

Secretario de la División de Ciencias Económico y Sociales

Dra. Leticia María González Velásquez

Jefe del Departamento de Ciencias Económico Administrativas

Dra. Lidia Amalia Zallas Esquer

Jefe de Departamento de Ciencias Sociales 
Año 13.

Revista de Investigación

Núm. 33

Académica sin Frontera

ISSN: 2007-8870

http://revistainvestigacionacademicasinfrontera.com

Recibido el 22 de agosto de 2020. Dictaminado mediante arbitraje favorablemente 18 de noviembre de 2020.

\section{Comité Directivo}

\section{Editor Responsable}

Dr. Francisco Espinoza Morales

Directora

Dra. Leticia María González Velásquez

Subdirector

Dr. Javier Carreón Guillen

Editor Científico

Dr. Cruz García Lirios

Master Gráfico

M.T.I. Francisco Alan Espinoza Zallas

Nos complace anunciar que su diario, "Academic Research Journal Withoutborders" (ISSN/EISSN 2007-8870) fue evaluado positivamente en la indexación Citefactor, ahora la página de la revista está disponible en línea, en caso de cualquier problema.

Journals Master | International Innovative Journal Impact Factor (IIJIF)

Red Latinoamericana de revistas Académicas en Ciencias Sociales y Humanidades
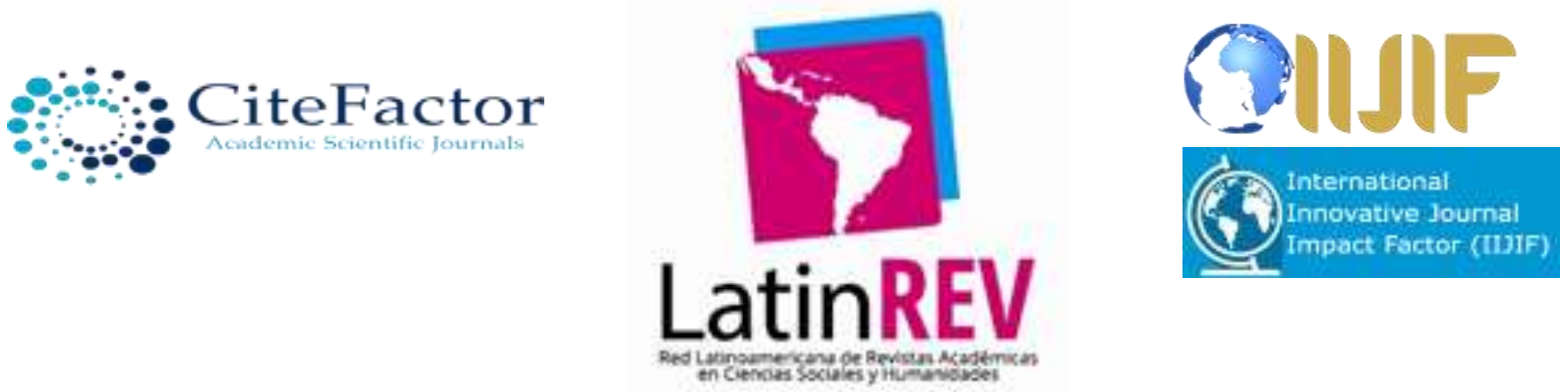
Año 13.

Revista de Investigación

Núm. 33

Académica sin Frontera

ISSN: 2007-8870

\section{http://revistainvestigacionacademicasinfrontera.com}

Recibido el 22 de agosto de 2020. Dictaminado mediante arbitraje favorablemente 18 de noviembre de 2020.

\section{Comité editorial}

Dra. Angélica María Rascón Larios

Universidad de Sonora. México

Dra. María del Rosario Molina González

Universidad de Sonora

Dra. Francisca Elena Rochin Wong

Universidad de Sonora. México

Dra. Lidia Amalia Zallas Esquer

Universidad de Sonora. México

Dra. Beatriz Llamas Arechiga

Universidad de Sonora. México

Dr. Rogelio Barba Álvarez

Universidad de Guadalajara. México

Dra. Rosa María Rincón Ornelas

Universidad de Sonora. México

Dr. Juan Flores Preciado

Universidad de Colima. México

Dr. Amado Olivares Leal. Universidad de Sonora

Universidad de Sonora. México

Dr. Guillermo Velázquez Valadez.

Instituto Politécnico Nacional (IPN) México

Dr. Hugo Nefstalí Padilla Torres.

Universidad Estatal de Sonora. México

Dr. Luis Ramón Moreno Moreno.

Universidad Autónoma de Baja California. México

Dr. Miguel Ángel Vázquez Ruiz.

Universidad de Sonora. México 


\section{http://revistainvestigacionacademicasinfrontera.com}

Recibido el 22 de agosto de 2020. Dictaminado mediante arbitraje favorablemente 18 de noviembre de 2020.

Dra. Lorena Vélez García.

Universidad Autónoma de Baja California. México

Dra. Pabla Peralta Miranda.

Universidad Simón Bolívar, Barranquilla, Colombia

Mtro. Roberto Espíritu Olmos

Universidad de Colima (FCA Tecomán) Colima

Dr. Héctor Priego Huertas.

Universidad de Colima (FCA Tecomán) Colima

Mtra. María Guadalupe Alvarado Ibarra.

Universidad de Sonora. México.

MSc. Celso Germán Sánchez Zayas

Universidad de Camagüey, Ignacio Agramonte Loynaz, Cuba

Dra. María Luisa Quintero Soto

Universidad Autónoma del Estado de México

Dr. Eyder Bolivar Mojica

Universidad Católica, Luis Amigó, Medellin, Colombia

Revisores de Textos en Inglés

Mtro. Renato Encinas

Mtra. Cecilia Guadalupe Martínez Solano

\section{Comité científico}

Dr. Rosendo Martínez Jiménez. Universidad Autónoma Benito Juárez de Oaxaca.

Dr. Hugo Neftalí Padilla. Universidad Estatal de Sonora

Dra. María Teresa Gaxiola Sánchez. Universidad de Sonora.

Dr. José Cesar Kaplan. Universidad Estatal de Sonora.

Dr. Alfredo Islas Rodríguez. Universidad de Sonora

Frecuencia de publicación: semestral / 2 números por año. 


\section{http://revistainvestigacionacademicasinfrontera.com}

Recibido el 22 de agosto de 2020. Dictaminado mediante arbitraje favorablemente 18 de noviembre de 2020.

Revista de Investigación Académica sin Frontera (RIASF) con (ISSN: 2007-8870) es un interlocutor internacional de acceso abierto revisado diario en línea en el ámbito del de las Ciencias Económicas Administrativas y Sociales. Su objetivo principal es dar a los trabajos de investigación de calidad. Cubre todas las sub-campos de los campos anteriormente mencionados. Proporciona la plataforma a académicos, estudiantes y profesionales. Sólo pública trabajos de investigación y artículos de revisión inicial. Documento presentado debe cumplir con algunos criterios como, debe ser original, inédita y no estén sometidos a ninguna otra revista.

RIASF es una revista arbitrada / Revisión por pares International. Publicamos documentos sobre una variedad de temas, contextos y estrategias de análisis que examinan la relación entre la rápida evolución para la Sociedad y la tecnología del conocimiento.

REVISTA DE INVESTIGACIÓN ACADÉMICA SIN FRONTERA, Año 13, No. 33, Julio - diciembre 2020, es una publicación semestral de investigación científica, editada por la Universidad de Sonora, a través de las División de Ciencias Económicas y Sociales, de la Unidad Regional Sur, Blvd. Lázaro Cárdenas No. 100, Col. Francisco Villa, Navojoa, Sonora, Sonora, México, C.P. 85880. Tel. (642) 42599-54.

http://www.revistainvestigacionacademicasinfrontera.com/, revistaacademicasinfrontera@ unison.mx. Editor responsable: Francisco Espinoza Morales. Reserva de Derechos al Uso Exclusivo: 042013-121811323700-203 e ISSN: 2007-8870, ambos otorgados por el Instituto Nacional de Derecho de Autor. Inscrita en el Directorio de LATINDEX, con Núm. De folio 20014, folio único 14590. Responsable de la última actualización de este Número, Unidad Informática de la Universidad de Sonora, fecha de la última modificación, 30 de diciembre 2020, indexada a Cite Factor Academic Scientific Journal y Journals Master (IIJIF) y Red Latinoamericana de Revistas Académicas en Ciencias Sociales y Humanidades, (Latín Rev). Las opiniones expresadas por los autores no necesariamente reflejan la postura del editor de la publicación. Se autoriza la reproducción total o parcial de los contenidos e imágenes en la presente publicación siempre y cuando se cuente con la autorización del editor y se cite plenamente la fuente. 
Año 13.

Revista de Investigación

Núm. 33

Académica sin Frontera

ISSN: 2007-8870

http://revistainvestigacionacademicasinfrontera.com

Recibido el 22 de agosto de 2020. Dictaminado mediante arbitraje favorablemente 18 de noviembre de 2020.

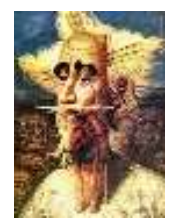

Nos complace anunciar que su diario, "Academic Research Journal Withoutborders" (ISSN/EISSN 2007-8870) fue evaluado positivamente en la indexación Citefactor, ahora la página de la revista está disponible en línea, en caso de cualquier problema.

Journals Master | International Innovative Journal Impact Factor (IIJIF)

Red Latinoamericana de revistas Académicas en Ciencias Sociales y Humanidades
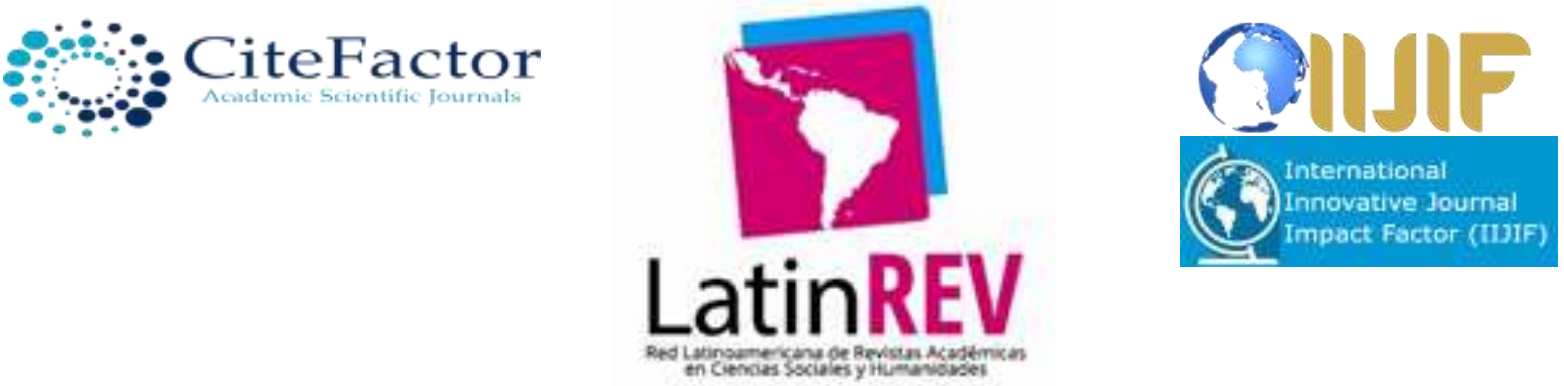

https://www.neliti.com

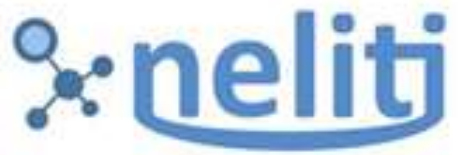

Indonesia's Research Repository
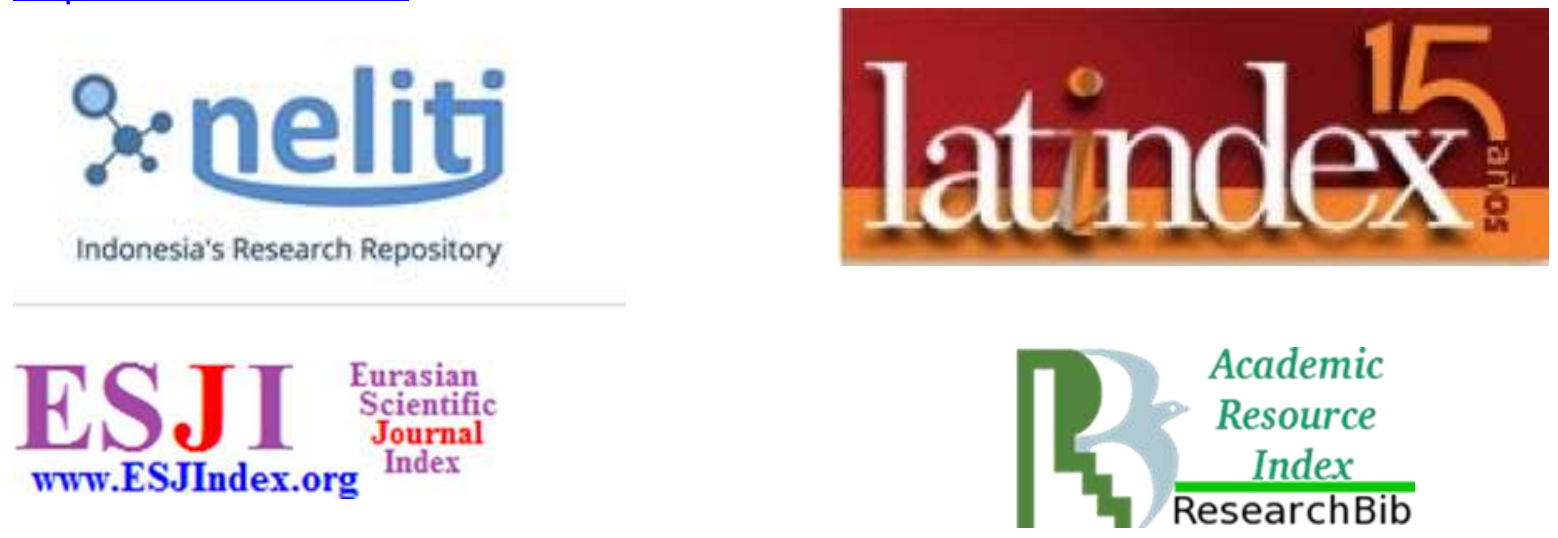


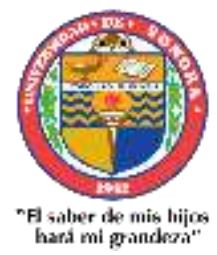

Año 13.

Núm. 33
( Julio - Diciembre 2020)

Revista de Investigación

Académica sin Frontera

ISSN: 2007-8870

http://revistainvestigacionacademicasinfrontera.com

Recibido el 22 de agosto de 2020. Dictaminado mediante arbitraje favorablemente 18 de noviembre de 2020. 